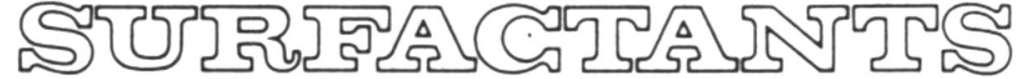

\section{DITMRG JNTS}

Zeitschrift für Physik, Chemie und Anwendung grenzflächenaktiver Stoffe

\section{Organschaft}

Die Zeitschrift ist das offizielle Organ der Fachgruppe "Waschmittelchemie" der Gesellschaft Deutscher Chemiker und des Deutschen Ausschusses für Grenzflächenaktive Stoffe im Verband der Chemischen Industrie e. V.

\section{Herausgeber}

Professor Dr. rer. nat. Bernhard Fell

\section{Schriftleitung}

Dr. rer. nat. Ulrich Buller (verantwort lich) und Dipl.-Chem. Barbara Buller Moosfeldstraße 10c, D-8067 Petershausen, Telefon: 0 8137/5817

\section{Wissenschaftlicher Beirat}

$H$. Andree, Düsseldorf

F. Asinger, Aachen

K. Blomeyer, Strombeek,

Belgien/Belgium

H. Casotti, Florida, Argentinien/

Argentinia

H. Distler, Ludwigshafen

W. Frank, Frankfurt

A. Hettche, Ludwigshafen

H. Hoffmann, Bayreuth

K. Kosswig, Marl

W. P. Meier, Schwalbach a. Ts.

A. W. Neumann, Toronto,

Ontario/ Canada

N. M. van Os, Amsterdam

Niederlande/Netherlands

M. J. Schick, N. Y./USA

H. Stache, Marl

\begin{tabular}{|c|c|}
\hline Kurzfassungen & 231 \\
\hline \multicolumn{2}{|l|}{ Anwendung } \\
\hline S. Willemse: Dosiergefäße für granulierte Waschmittel & 234 \\
\hline $\begin{array}{l}\text { D. Vollhardt: Sprühablagerungen von Pflanzenschutzmitteln ..... } \\
\text { V. Hayden, P. Hodul: Eigenschaften von Schaumsystemen für die }\end{array}$ & 239 \\
\hline Knitterfreiausrüstung von Baumwollgeweben . . . . . . . . . . . & 243 \\
\hline
\end{tabular}

\section{Physikalische Chemie}

A. Georgieva, R. Detscheva: Wirkung von Caprolactamlösungen beim Färbeprozeß mit Dispersionsfarbstoffen . . . . . . . . . . .

K. N. Mehrotra, M. Sharma, A. S. Gahlaut: Infrarotspektroskopische sowie röntgenspektroskopische Messungen und Thermoanalyse an Praseodymseifen.

J. Zakrzewski: Verallgemeinerung des Bailey'schen Verteilungsgesetzes

Th. El-Nabarawy, N. H. Amin: Adsorption von kationischen Tensiden an Kohleoberflächen im Vergleich zu Sauerstoffkomplexen. R. P. Varma, A. Kumar: $\mathrm{pH}-$ Wert-Bestimmungen und Messungen zur Solubilisierung von Erdalkalibutyraten

R. Wüstneck, R. Miller, G. Czichocki: Charakterisierung des Adsorptionsverhaltens von Natrium-n-Alkylsulfaten an der Grenzfläche Luft/wäßrige Lösung mit Hilfe verschiedener Adsorptionsisothermen.

\section{Synthese}

J. Beger, R. Jacobi, U. Süßmitt, T. Müller, A. Stöhr, V. Schmidt: Mehrfunktionelle N-Tenside; Teil XIII

D. N. Bhattacharyya, R. S. Iyer: Ein W/O-Emulgator aus Fettsäuren des Leinsamenöls.

J. Pernak, J. Jędraszczyk, A. Skrzypczak, J. Krysiński, A. Wẹcras: Antimikrobielle Wirkung von Iminiumverbindungen .

\section{Analyse}

W. Gerhardt t, G. Czichocki, H.-R. Holzbauer, C. Martens,

B. Weiland: Zur Synthese und Analytik von Ethercarbonsäuren; Teil II 285

M. Sak-Bosnar, Lj. Zelenka, M. Budimir: Bestimmung von Schwefelsäure und Alkylbenzensulfonsäure

\section{Geräteinformationen}

Pestizide schnell bestimmen. . . . . . . . . . . . . . . . 245

Spektralphotometer mit hohem Leistungsvermögen . . . . . . . . . 252

Vollautomatische c.m.c.-Bestimmung ................. 252

EOX-Kennzahlen vollautomatisch bestimmen ............ 256

Umwälzkühler für Einzelplatzlösungen. . . . . . . . . . . . . . . . . . . 264

Flüssigkeits-Festkörper-Extraktor benutzt Standard-Soxhlet-Glaswaren ............................ 270

Gaschromatographie-System mit hohem Probendurchsatz . . . . . 270

Computergesteuerte Labor-Rührwerkskugelmühle . . . . . . . . . . . 277

Automatische $\mathrm{pH}$-Titration zum günstigen Preis . . . . . . . . . . . . . . 277

Korngrößenanalyse im Bereich 0,04 bis $1000 \mu \mathrm{m} \ldots . . \ldots . . .291$

\begin{tabular}{lc}
\hline Patente & 292 \\
\hline Neuigkeiten & 297 \\
\hline Termine & 299 \\
\hline Impressum & 300
\end{tabular}




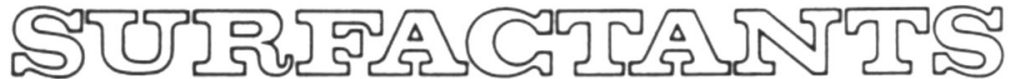
DIM IMGSRTS

Journal for Theory, Technology and Application of Surfactants

\section{Organ}

The journal is the official organ of the special section "Waschmittelchemie" of the Gesellschaft Deutscher Chemiker and of the Deutscher Ausschuß für Grenzflächenaktive Stoffe im Verband der Chemischen Industrie e.V.

\section{Editor}

Professor Dr. rer. nat. Bernhard Fell

\section{Managing Editor}

Dr. rer. nat. Ulrich Buller (responsible) and Dipl.-Chem. Barbara Buller, Moosfeldstr. 10c, D-8067 Petershausen, Phone: $08137 / 5817$

\section{Specialist Advisors}

H. Andree, Düsseldorf

F. Asinger, Aachen

K. Blomeyer, Strombeek,

Belgien/Belgium

H. Casotti, Florida, Argentinien/

Argentinia

$\mathrm{H}$. Distler, Ludwigshafen

W. Frank, Frankfurt

A. Hettche, Ludwigshafen

H. Hoffmann, Bayreuth

K. Kosswig, Marl

W. P. Meier, Schwalbach a. Ts.

A. W. Neumann, Toronto,

Ontario/Canada

N. M. van Os, Amsterdam,

Niederlande/Netherlands

M. J. Schick, N. Y./USA

H. Stache, Marl

\section{Summaries}

231

Application

S. Willemse: Dispensing Devices for Granular Detergents 234

D. Vollhardt: Spray Pattern for Plant Protection

V. Hayden, P. Hodul: Properties of Foaming Systems for Crease Resistant Finishes for Cotton Fabrics

\section{Physical Chemistry}

A. Georgieva, R. Detscheva: Effect of Caprolactam on Dyeing with Dispersive Dyes

K. N. Mehrotra, M. Sharma, A. S. Gahlaut: Infrared, X-Ray and Thermal Analysis of Praseodym Soaps

J. Zakrzewski: Generalization of Bailey's Random Distribution Rule.

Th. El-Nabarawy, N. H. Amin: Adsorption of Cationic Surfactants in Relation to Oxygen Complexes on Carbons

R. P. Varma, A. Kumar: $\mathrm{pH}$ Determinations and the Solubilizing Efficiency of Alkaline Earth Metal Butyrates.

R. Wüstneck, R. Miller, G. Czichocki: Characterization of the Adsorption Behaviour of Sodium-n-Alkylsulphates at the Interface Air/Aqueous Solution by Different Adsorption Isotherms

\section{Synthesis}

J. Beger, R. Jacobi, U. Süßmitt, T. Müller, A. Stöhr, V. Schmidt: Multifunctional N-Surfactants; Part XIII

D. N. Bhattacharyya, R. S. Iyer: Water-in-Oil Emulsifier from Bodied Linseed Oil Fatty Acids

J. Pernak, J. Jẹdraszczyk, A. Skrzypczak, J. Krysiński,

A. Wẹcras: Antibacterial Effects of Imidazolium Compounds

\section{Analysis}

W. Gerhardt †, G. Czichocki, H.-R. Holzbauer, C. Martens, B. Weiland: Synthesis and Analysis of Ether Carboxylic Acids; Part II 285 M. Sak-Bosnar, Lj. Zelenka, M. Budimir: Determination of Sulfuric and Alkylbenzensulfonic Acid.

\section{Apparatus Informations}

Fast Determination of Pesticides. . . . . . . . . . . . . . . . . 245

Spectrophotometer with High Efficiency . . . . . . . . . . . . . . 252

Fully Automatic c. m.c. Determination ................. 252

Fully Automated Determination of EOX Charateristics ......... 256

Circulating Cooler for Single-place Solutions . . . . . . . . . . . 264

Liquid-Solid-Extractor Using Standard Soxhlet Glassware . . . . . . 270

Gas Chromatography System with a High Sample Throughput . . . 270

Computer-controlled Agitator Ball Mill for Laboratories . . . . . . . . 277

Automated pH Titrations at a Favorable Price . . . . . . . . . . . 277

Granulometric Analysis in the Range from 0.04 to $1000 \mu \mathrm{m}$. . . . 291

\begin{tabular}{ll}
\hline Patents & 292 \\
\hline News & 297 \\
\hline Dates & 299 \\
\hline Imprint & 300
\end{tabular}

